\title{
Esclerose lateral amiotrófica: descrição de aspectos clínicos e funcionais de uma série de casos numa região de saúde do nordeste do Brasil
}

\section{Amyotrophic lateral sclerosis: description of clinical and functional characteristics of a series of cases in a Northeast health region of Brazil}

\author{
Leandro Pereira Silva ${ }^{1}$, Cristiane Aguiar Gusmão ${ }^{2}$, Karla Rocha Pithon², Thaís Bitencourt Peixoto Gomes ${ }^{1}$, Elzo Pereira Pinto \\ Junior ${ }^{3}$ \\ 1. Fisioterapeuta, graduado pela Universidade Estadual do Sudoeste da Bahia (UESB), Vitória da Conquista, BA, Brasil. 2. Docente curso de Fisioterapia da \\ Universidade Estadual do Sudoeste da Bahia (UESB), Vitória da Conquista, BA, Brasil. 3. Doutorando em Saúde Pública pelo Programa de Pós-Graduação em
} Saúde Coletiva do Instituto de Saúde Coletiva - Universidade Federal da Bahia (ISC-UFBA), Salvador, BA, Brasil

\section{Resumo}

Introdução: A Esclerose Lateral Amiotrófica (ELA) é uma doença neurodegenerativa que provoca grandes prejuízos na funcionalidade dos indivíduos acometidos. Objetivo: descrever características sociodemográficas, clínicas e funcionais de uma série de casos diagnosticados com Esclerose Lateral Amiotrófica em uma região de saúde do Estado da Bahia. Métodos: trata-se do relato de uma série de casos de ELA, identificados na microrregião de Saúde de Jequié, circunscrita à 13a Diretoria Regional de Saúde (DIRES) da Bahia. Foram incluídos indivíduos com diagnóstico médico de ELA, comprovado por exame de Eletroneuromiografia, independente do sexo ou idade. Após a identificação dos sujeitos, foram realizadas visitas domiciliares para coleta de dados sociodemográficas, sintomas neurológicos e avaliação funcional. Resultados: A média de idade dos seis participantes da pesquisa foi 52,8 anos (DP $\pm 6,3$ anos), tendo os primeiros sintomas se manifestado a partir dos 46 anos de idade. Os principais sintomas da Esclerose Lateral Amiotrófica foram fraqueza $(50,0 \%)$ e dor $(50,0 \%)$ e durante a progressão foi observada a presença de fraqueza $(100,0 \%)$, seguida de disartria $(83,3 \%)$, fasciculações $(66,7 \%)$ e tremor (66,7\%). De modo geral, as atividades que os sujeitos referiram realizar com mais frequência foram "vestir-se da cintura para baixo" (100,0\%) e alimentação $(83,3 \%)$. Conclusão: Os achados desta pesquisa apontam a necessidade de ofertar um conjunto de serviços de saúde para os indivíduos com ELA, em uma perspectiva interdisciplinar, que envolve o acompanhamento médico-farmacológico, nutricional, assistência em fisioterapia, fonoaudiologia e terapia ocupacional, além do acompanhamento psicológico para os pacientes e suas famílias.

Palavras-chave: Esclerose Lateral Amiotrófica. Relatos de Casos. Manifestações Neurológicas. Atividades Cotidianas

\begin{abstract}
Introduction: Amyotrophic Lateral Sclerosis (ALS) is a neurodegenerative disease that causes great damages in the functionality of affected individuals. Objective: To describe sociodemographic, clinical and functional characteristics of a series of cases diagnosed with Amyotrophic Lateral Sclerosis. Methods: This is a report of a series of cases of ALS, identified in the Jequié Health Micro-region, which is limited to the 13th Regional Health Directorate (DIRES) of Bahia. We included individuals with a medical diagnosis of ALS, confirmed by electromyography, independent of gender or age. After identification of the subjects, home visits were made to collect sociodemographic data, neurological symptoms and functional evaluation. Results: The mean age of the six study participants was 52.8 years ( $\mathrm{SD} \pm 6.3$ years), with the first symptoms occurring after the age of 46 years. The main symptoms of Amyotrophic Lateral Sclerosis were weakness (50.0\%) and pain (50.0\%) and during the progressive weakness was observed (100.0\%), followed by dysarthria (83.3\%), fasciculations (66.7\%) and tremor (66.7\%). In general, the activities which the subjects reported to execute most frequently were "dressing from the waist down" (100.0\%) and feeding (83.3\%). Conclusion: The findings of this research point to the need of offer a set of health services for individuals with ALS, in an interdisciplinary perspective, involving medical-pharmacological, nutritional, physical therapy, phonoaudiology and occupational therapy besides psychological counseling for patients and their families.
\end{abstract}

Key words: Amyotrophic Lateral Sclerosis. Case Reports. Neurologic Manifestations. Activities of Daily Living.

INTRODUÇÃO

A Esclerose Lateral Amiotrófica (ELA) é uma doença neurodegenerativa de etiologia desconhecida e se caracteriza pelo acometimento tanto do neurônio motor superior quanto do inferior. A ELA atinge progressivamente as células do núcleo motor dos nervos cranianos do tronco encefálico, do corno anterior da medula e das vias corticoespinhais e corticobulbares, resultando em paralisia motora progressiva e irreversível ${ }^{1,2,3}$.

Embora a Esclerose Lateral Amiotrófica seja uma doença rara, relatos apresentam descrição de pacientes com tal patologia desde o início do século XX; entretanto o aumento de sua incidência nas últimas décadas tem chamado atenção da população e estudiosos, tornando-se alvo de campanhas de comunicação em massa e de investigações mais robustas sobre aspectos clínicos e epidemológicos ${ }^{1}$. Recente revisão sistemática sobre a epidemiologia da Esclerose Lateral Amiotrófica encontrou uma incidência mundial relativamente uniforme, variando de 0,31 (Hong Kong) a 3.2/100.000 habitantes (Limousine- França) ${ }^{4}$. Especificamente no contexto Europeu, a incidência estimada de ELA foi de 2.16/100.000 
habitantes, sendo os maiores valores observados em homens ${ }^{5}$. No Brasil, ainda são escassas as estimativas nacionais sobre essa patologia, sendo que um dos principais estudos de abrangência nacional identificaram 443 casos dessa doença no País ${ }^{6}$.

Um conjunto de variáveis tem sido investigado como possíveis fatores de risco para a Esclerose Lateral Amiotrófica, considerando desde causas genéticas até fatores ambientais ${ }^{7}$. Entretanto, cerca de $90 \%$ dos casos são considerados esporádicos e com etiologia desconhecida ${ }^{8}$. Apesar dessas incertezas quanto à causa da ELA, parece haver consenso que a idade do indivíduo guarda forte relação com o aparecimento da doença, sendo considerada um preditor, haja vista que as prevalências são maiores em sujeitos com idade de 55 a 75 $\operatorname{anos}^{1,9}$.

Clinicamente, a maioria das pessoas acometidas por ELA inicialmente relatam fraqueza muscular assimétrica e fasciculações. Durante a progressão da doença começam a surgir sintomas de dor, disfagia e disartria. Com o avanço da doença, os indivíduos passam a ter maior comprometimento funcional, com declínio de sua capacidade para a realização de atividades básicas da vida diária, que em última instância, os torna dependentes dos cuidados de terceiros. A média de sobrevida desses pacientes varia de 2 a 5 anos após o início dos primeiros sintomas e a causa de óbito mais frequente nesses indivíduos é a insuficiência respiratória, associada à disfagia e à broncoaspiração $0^{10,11}$.

Embora a ELA seja uma doença ainda sem cura, segundo o protocolos clínicos e diretrizes terapêuticas têm sido desenvolvidas para tentar garantir qualidade de vida e aumento da sobrevida a esses pacientes, especialmente com o uso de medicamentos específicos, como o Riluzol ${ }^{12}$. Considerando a gravidade da patologia e a multiplicidade de manifestações clínicas, faz-se necessário a participação de uma equipe multidisciplinar de saúde bem articulada e capacitada que possa tomar as medidas paliativas e atenuar o sofrimento do indivíduo ${ }^{13,14}$.

Esta pesquisa teve como objetivo descrever características sociodemográficas, clínicas e funcionais de uma série de casos diagnosticados com ELA numa região de saúde do Estado da Bahia, em 2014.

\section{DISCUSSÃo}

Este estudo trata-se do relato de uma série de casos de Esclerosa Lateral Amiotrófica, identificados na microrregião de Saúde de Jequié, circunscrita à 13a Diretoria Regional de Saúde (DIRES) do Estado da Bahia. Jequié é um município de aproximadamente 150 mil habitantes, sede da 13a DIRES, localizado na região sudoeste da Bahia, distante $365 \mathrm{~km}$ de Salvador, capital do Estado.

Após aprovação deste estudo pelo Comitê de Ética em Pesquisa (CEP), conforme a Resolução 466/2012 do conselho Nacional de Saúde, sob o parecer n. 805545, os pesquisadores deram início à busca dos indivíduos com diagnóstico de ELA. Para identificar tais indivíduos, foram consultados os registros de pacientes da 13a DIRES, do Hospital Geral Prado Valadares (HGPV), maior hospital público e com leitos para pacientes neurológicos na região, além de visita às clinicas de Neurologia no município sede. O HGPV localiza-se em Jequié, é um hospital regional de Sistema Único de Saúde, servindo de referência para aproximadamente 30 municípios, caracterizando-se como o principal serviço de assistência neurológica para casos mais graves. Já a $13^{\circ}$ Diretoria Regional de Saúde é o local onde são distribuídos medicamentos gratuitos utilizados pelos pacientes acometidos por Esclerose Lateral Amiotrófica.

Os critérios de inclusão para compor a amostra de casos de Esclerose Lateral Amiotrófica foram: indivíduos com diagnóstico médico de ELA, comprovado por exame de Eletroneuromiografia, independente do sexo ou idade. Foram excluídos os sujeitos que não residiam nos municípios assistidos pela 13a DIRES, que já haviam falecidos ou que não foi possível identificar o endereço para a visita e coleta de dados.

Após a visita aos três tipos de serviços, foram encontrados os registros de 10 pacientes com diagnóstico de Esclerose Lateral Amiotrófica, sendo um deles proveniente do HGPV, um de clínicas particulares e oito da 13a DIRES. Dos oito pacientes registrados na DIRES, apenas quatro foram encontrados para realização da entrevista, haja vista a ocorrência de dois óbitos e em outros dois casos, a documentação e prontuário não estavam completos, de modo que não foi possível localizá-los (Figura 1).

Figura 1. Organograma de recrutamento dos participantes da pesquisa.

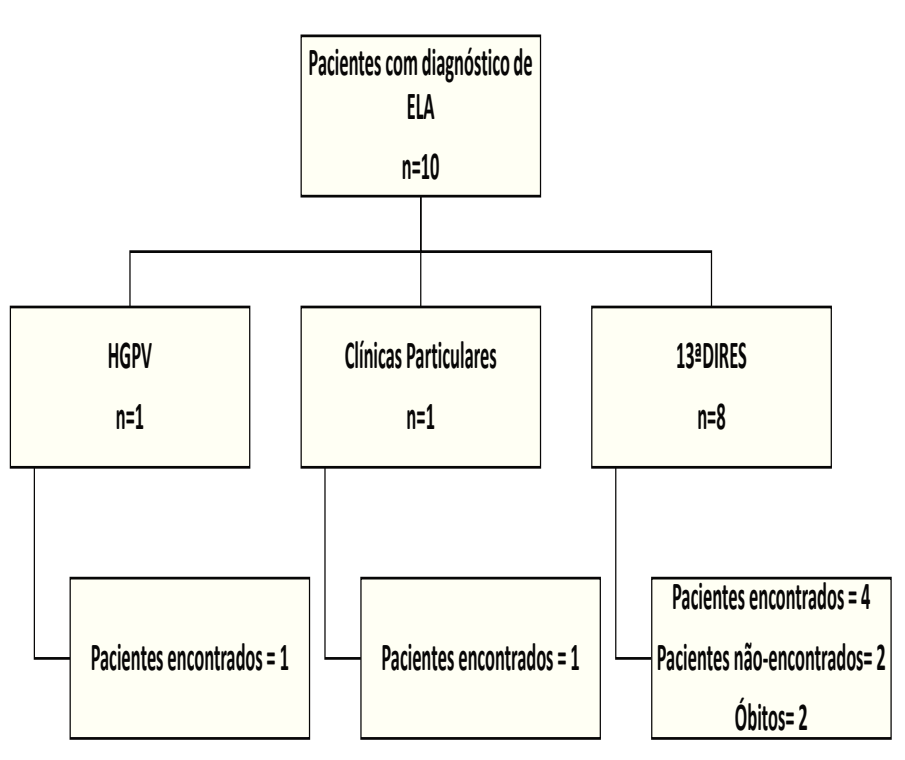


Após a identificação dos sujeitos, foram realizadas visitas domiciliares para aplicação de um questionário com o intuito de obter informações sobre características sociodemográficas, sintomas neurológicos e progressão da Esclerose Lateral Amiotrófica. No questionário, optou-se por utilizar uma versão adaptada da escala de Katz para avaliar a dependência funcional dos sujeitos na realização das atividades básicas da vida diária (ABVD). A entrevista foi conduzida preferencialmente com o paciente; no entanto, caso ele não tivesse condições de responder, o cuidador ou familiar responsável era solicitado a responder. Antes da realização da entrevista, os pesquisadores entregaram o Termo de Consentimento Livre e Esclarecido (TCLE), e após a sua assinatura, teve início a entrevista.

Após a coleta de dados, os dados foram tabulados em planilhas de texto Excel, da Microsoft. Por se tratar de uma amostra pequena, optou-se por representar alguns dos resultados em quadros, indicando as variáveis de cada um dos seis pacientes. Em outros casos, foram construídas tabelas. A análise dos dados contou com elementos da estatística descritiva, com cálculo da frequência absoluta e relativa, e foi realizada no software "Social Package for the Social Sciences - SPSS", versão 17.0

\section{RESULTADOS}

A média de idade dos seis participantes da pesquisa foi 52,8 anos (Desvio Padrão $\pm 6,3$ anos), tendo os primeiros sintomas se manifestado a partir dos 46 anos de idade. Em relação ao período de tempo entre a ocorrência dos primeiros sintomas e o diagnóstico da ELA foi possível notar uma variação de 1 a 28 meses (Quadro 1).

Quadro 1. Descrição dos casos de ELA, segundo idade atual, idade do início dos sintomas e diferença de tempo entre os primeiros sintomas e o diagnóstico.

\begin{tabular}{|lrrr|}
\hline Paciente & Idade atual (anos) & $\begin{array}{r}\text { Idade do início dos sintomas } \\
\text { (anos) }\end{array}$ & $\begin{array}{r}\text { Tempo entre o início dos sintomas e o } \\
\text { diagnóstico (meses) }\end{array}$ \\
\hline A & 52 & 51 & 12 \\
B & 49 & 46 & 19 \\
C & 49 & 47 & 01 \\
D & 54 & 53 & 06 \\
E & 48 & 47 & 04 \\
F & 65 & 62 & 28 \\
Média (DP*) & $52,8( \pm 6,3)$ & $51( \pm 6,0)$ & $11,6( \pm 10,2)$ \\
\hline
\end{tabular}

*DP : Desvio-Padrão

Os principais sintomas associados à Esclerose Lateral Amiotrófica relatados pelos sujeitos desta pesquisa foram fraqueza $(50,0 \%)$ e dor $(50,0 \%)$, seguidos de tremores $(33,3 \%)$ e dormência (33,3\%). Durante a progressão da doença, todos os entrevistados relataram a presença de fraqueza $(100,0 \%)$, seguido de disartria $(83,3 \%)$, fasciculações $(66,7 \%)$ e tremor $(66,7 \%)$. Os sintomas menos comuns foram perda sensorial $(16,7 \%)$, incontinência $(16,7 \%)$ e tonturas $(16,7 \%)$ (Tabela 1).

Tabela 1. Sintomas apresentados pelos indivíduos com diagnóstico de ELA, no início e durante a progressão da doença.

\begin{tabular}{lrrrr}
\hline \multirow{2}{*}{ Sintomas } & \multicolumn{3}{r}{ Inicio } & \multicolumn{2}{r}{ Progressão } \\
\cline { 2 - 6 } & $\mathbf{n}$ & $\mathbf{\%}$ & $\mathbf{n}$ & \% \\
\hline Fraqueza & 3 & 50,0 & 6 & 100,0 \\
Disartria & 1 & 16,7 & 5 & 83,3 \\
Fasciculações & 1 & 16,7 & 4 & 66,7 \\
Tremor & 2 & 33,3 & 4 & 66,7 \\
Disfagia & - & - & 3 & 50,0 \\
Dor & 3 & 50,0 & 3 & 50,0 \\
\hline
\end{tabular}

\begin{tabular}{lrrrr}
\hline \multirow{2}{*}{ Sintomas } & \multicolumn{2}{r}{ Inicio } & \multicolumn{2}{r}{ Progressão } \\
\cline { 2 - 5 } & $\mathbf{n}$ & \% & $\mathbf{n}$ & \% \\
\hline Desconforto respiratório & - & - & 3 & 50,0 \\
Atrofia & - & - & 3 & 50,0 \\
Câimbras & - & - & 2 & 33,3 \\
Dormência & 2 & 33,3 & 2 & 33,3 \\
Sinal de Babinski & - & - & 2 & 33,3 \\
Perda sensorial & - & - & 1 & 16,7 \\
Incontinência & - & - & 1 & 16,7 \\
Tonturas & - & - & 1 & 16,7 \\
\hline
\end{tabular}

A análise dos aspectos funcionais, avaliados a partir da realização das atividades básicas da vida diária, demonstrou grandes diferenças entre os participantes da pesquisa, enquanto o paciente $B$ conseguiu realizar todas as atividades, os pacientes A e $C$ conseguiram realizar apenas três delas. De modo geral, as atividades que os sujeitos referiram realizar com mais frequência foram "vestir-se da cintura para baixo" $(100,0 \%)$ e alimentação (83,3\%), enquanto "transferência no leito" $(33,3 \%)$ representou a ABVD com menor frequência de realização. 
Quadro 2. Avaliação funcional para realização de Atividades Básicas da Vida Diária em indivíduos com diagnóstico de ELA.

\begin{tabular}{|c|c|c|c|c|c|c|}
\hline Função & Pct.* A & Pct.* B & Pct. $* \mathrm{C}$ & Pct.* D & Pct.* E & Pct. $* \mathrm{~F}$ \\
\hline Marcha & & $\mathrm{X}$ & & $x$ & & $\mathrm{X}$ \\
\hline Subir escadas & & $\mathrm{x}$ & & $x$ & $x$ & $\mathrm{X}$ \\
\hline Alimentação & $x$ & $x$ & $x$ & $x$ & $x$ & \\
\hline Higiene pessoal & & $x$ & & $x$ & $x$ & $x$ \\
\hline Transferência no leito & & $x$ & & $x$ & & \\
\hline Sedestação para ortostase & & $\mathrm{X}$ & & $x$ & & $X$ \\
\hline Vestir-se cintura $\mathrm{p} /$ cima & $x$ & $x$ & $\mathrm{X}$ & $x$ & & \\
\hline Vestir-se cintura $\mathrm{p} /$ baixo & $x$ & $x$ & $x$ & $x$ & $x$ & $x$ \\
\hline
\end{tabular}

*Pct: Paciente

\section{DISCUSSÃO}

Os achados desta pesquisa revelaram poucos casos de ELA em uma região de saúde do Nordeste do Brasil, o que confere a essa patologia o status de doença rara. Tais resultados corroboram a produção científica tanto em nível nacional quanto internacional, que apontam baixas incidências e prevalências. Recente metanálise que revisou 44 estudos de base populacional identificou 13.146 casos de Esclerose Lateral Amiotrófica em 45 áreas geográficas distintas no mundo, que correspondia a uma população de 825 milhões de pessoas, sendo estimada uma incidência mundial de 1,68 casos/100.000 habitantes ${ }^{15}$. Outros estudos de menor abrangência encontraram valores semelhantes na República do Chipre $(1,26 / 100.000)^{16}$, no Sudestes da Alemanha (2,4/100.000 habitantes) ${ }^{17}$, e na cidade de Buenos Aires, Argentina (1,04/100.000 habitantes) ${ }^{18}$. Além desses dados de estudos já realizados, estimativas apontam que a ocorrência de ELA irá aumentar 69\% de 2015 até 2040, saltando de 222.801 para 367.674 casos $^{19}$.

O diagnóstico de ELA costuma acontecer em indivíduos adultos, acima dos 40 anos de idade, tal como foi observado nesta série de casos. Esses achados estão em consonância com uma revisão sistemática de estudos clínicos brasileiros, que mostraram que a média de idade do início dos sintomas foi aos 55 anos $^{20}$, valor inferior aos relatados em outros países europeus e norteamericanos, que indicam o surgimento dos primeiros sinais da doença após os 59 anos $^{5,21,22}$.

Apesar dos recentes avanços biomédicos, o diagnóstico da Esclerose Lateral Amiotrófica ainda é um desafio para os serviços de saúde, o que implica, em alguns casos, a um longo período de espera entre o início dos sintomas e a correta definição de caso. Os resultados deste trabalho se assemelham com os dados de outras investigações, que estimam em 14 meses o tempo médio para a conclusão do diagnóstico de ELA ${ }^{23}$. Este longo tempo de espera para o diagnóstico impacta negativamente no cuidado aos indivíduos acometidos com essa doença, tendo em vista o atraso para o início do tratamento adequado.

Os sujeitos participantes desta investigação apresentaram como principais sintomas inicias a fraqueza e a dor, resultados que também são evidenciados em outros estudos realizadas tanto no Brasil como em outros países ${ }^{16,18}$. No tocante à evolução do quadro clínico, as pesquisas apontam o comprometimento progressivo do sistema nervoso. Recente revisão indicou como os principais sintomas no curso da doença a piora do quadro de fraqueza, acometendo tanto membros inferiores quanto superiores, e o agravamento da disartria, fasciculações, disfagia, dor, câimbras, dormência, perdas sensoriais, culminando com os problemas de desconforto respiratório, cuja evolução costuma levar ao óbito ${ }^{24}$.

A evolução dos sintomas da Esclerose Lateral Amiotrófica impacta de modo bastante significativo à realização de atividades cotidianas nesses indivíduos, sejam elas atividades básicas ou instrumentais ${ }^{25}$, sendo que a dependência no desempenho dessas ações deve ser vista como importante desfecho clínico para guiar o cuidado ao paciente ${ }^{26}$. O presente estudo apontou que, dentre o que se considera um conjunto de atividades básicas da vida diária, as maiores limitações observadas nos pacientes com ELA foram relacionadas ao vestir-se, alimentar-se sozinho, realizar a higiene pessoal e subir escadas. As limitações na realização das atividades da vida diária também foram relatadas por Kehyayan e colaboradres ${ }^{27}$, que descreveram o perfil de 2.092 pacientes canadenses com ELA e identificaram que $54,9 \%$ desses indivíduos precisavam de assistência para desempenhar atividades cotidianas.

O cuidado ao paciente com Esclerose Lateral Amiotrófica deve compreender as diversas manifestações da doença no organismo do indivíduo, de modo que a reabilitação seja conduzida por equipes multiprofissionais e que observem tanto os aspectos físicos, quanto os psicológicos e sociais dos pacientes e de seus cuidadores ${ }^{28}$. No âmbito específico das intervenções relacionadas aos problemas funcionais, recente artigo de revisão da literatura apontou a importância de exercícios de flexibilidade, fortalecimento muscular e algumas atividades aeróbicas, e um enfoque terapêutico aos principais sintomas, como dor, espasticidade, câimbras, fadiga e dificuldade de 
eliminação de secreção ${ }^{25}$.

Esta investigação apresenta como uma de suas limitações o reduzido número de pacientes investigados, de modo que a pequena quantidade de indivíduos com o diagnóstico de ELA impede a generalização desses resultados para cenários mais ampliados. Além disso, outra limitação que merece ser destacada é a utilização de um instrumento mais genérico para a avaliação do desempenho das atividades da vida diária, já que ainda são escassos os instrumentos traduzidos e validados para investigar aspectos mais ampliados de saúde aos indivíduos com Esclerose Lateral Amiotrófica. Apesar dessas limitações, dada a incipiência de produção sobre essa temática, esse estudo é um dos primeiros a apresentar um perfil de uma série de casos de ELA, caracterizando as principais características clínicas e repercussões funcionais dessa doença em indivíduos que residem em um município do interior da Região Nordeste do Brasil.

Os resultados deste estudo com uma série de casos evidenciaram que a Esclerose Lateral Amiotrófica acometeu indivíduos adultos, acima dos 50 anos, com grandes variações entre o tempo do início dos sintomas e a conclusão do diagnóstico, sendo a fraqueza, disartria, fasciculações, tremores e dor os principais sintomas do início e progressão da doença.
Além disso, evidenciou-se ainda que a evolução desta patologia repercutiu em maiores dificuldade nas realização de atividades da vida diária, especialmente no vestir-se, alimentar-se sozinho e higiene pessoal.

Apesar das potencialidades dessa série de casos, sugerese o desenvolvimento de estudos com metodologias mais complexas. Surgem como possibilidades, estudos de casocontrole, para avaliar fatores associados à ocorrência da ELA, tendo em vista que se trata de um desfecho raro, ou estudos de coorte, a fim de avaliar a evolução dessa doença e fatores de prognóstico do óbito, que envolvendo o acompanhamento sistemático de casos já diagnosticados.

Os achados desta pesquisa apontam para a necessidade de ofertar um conjunto de serviços de saúde para os indivíduos com diagnóstico de ELA, em uma perspectiva interdisciplinar, que envolve o acompanhamento médico-farmacológico, nutricional, assistência em fisioterapia, fonoaudiologia e terapia ocupacional, além do acompanhamento psicológico para os pacientes e suas famílias. Nesse sentido, espera-se que os serviços públicos de saúde sejam capazes de atender de forma integral e resolutiva esses sujeitos, de modo a assegurar seu direito à saúde, como previsto nas leis do Sistema Único de Saúde.

\section{REFERÊNCIAS}

1. Van Es MA, Hardiman O, Chio A, Al-Chalabi A, Pasterkamp RJ, Veldink JH, et al. Amyotrophic lateral sclerosis. The Lancet. 2017 May. 390(10107): 2084-98. doi: http://dx.doi.org/10.1016/S0140-6736(17)31287-4.

2. Van der Graaff MM, de Jong JM, Baas F, de Visser M. Upper motor neuron and extra-motor neuron involvement in amyotrophic lateral sclerosis: A clinical and brain imaging review. Neuromuscul Disord. 2009 Jan;19(1):53-8. doi: 10.1016/j. nmd.2008.10.002.

3. Turner MR, Hardiman O, Benatar M, Brooks BR, Chio A, Carvalho M, et al. Controversies and priorities in amyotrophic lateral sclerosis. Lancet Neurol. 2013 Mar; 12(3):310-22. doi: 10.1016/S1474-4422(13)70036-X.

4. Chiò A, Logroscino G, Traynor BJ, Collins J, Simeone JC, Goldstein LA, et al. Global epidemiology of amyotrophic lateral sclerosis: A systematic review of the published literature. Neuroepidemiology. 2013; 41(2): 118-30. doi: 10.1159/000351153.

5. Logroscino G, Traynor BJ, Hardiman O, Chiò A, Mitchell D, Swingler RJ, et al. Incidence of amyotrophic lateral sclerosis in Europe. J Neurol Neurosurg Psychiatr. 2010 Apr; 81(4): 385-90. doi: 10.1136/jnnp.2009.183525.

6. Dietrich-Neto F, Calegaro D, Dias-Tosta E, Silva HA, Ferraz ME, LimaJMB, et al. Amyotrophic lateral sclerosis in Brazil: 1998 national survey. Arq Neuropsiquiatr. 2000; 58(3): 607-615. doi: http://dx.doi.org/10.1590/S0004$282 \times 2000000400002$

7. Cronin, S.; Hardiman, O.; Traynor, BJ. Ethnic variation in the incidence of ALS: a systematic review. Neurology. 2007 Mar; 68(13): 1002-7. doi: 10.1212/01. wnl.0000258551.96893.6f.

8. Clecio Godeiro Junior; Acary S.B. Oliveira; Andre C. Felicio; Marco A. Chieia; Alberto Alain Gabbai. Conjugal amyotrophic lateral sclerosis in Brazil. Arq. Neuro-Psiquiatr. 2009 Dec; 67(4): 1045-1048. doi: http://dx.doi.org/10.1590/ S0004-282X2009000600015.

9. Valadi N. Evaluation and management of Amyotrophic Lateral Sclerosis. Prim
Care. 2015 Jun; 42(2):177-87. doi: 10.1016/j.pop.2015.01.009.

10. Phukan, J.; Hardiman, O. The management of amyotrophic lateral sclerosis. J. Neurol. 2009 Feb; 256 (2): 86-176. doi: 10.1007/s00415-009-0142-9.

11. Ferguson TA, Elman LB. Clinical presentation and diagnosis de amyotrophic lateral sclerosis. Neuro Rehabilitation. 2007; 22(6):409-16.

12. Ghezzi SR, Fontes SV, Aguiar AS, Vitali LM, Fukujima MM, Ortensi FMF. Qualidade do sono de pacientes com esclerose lateral amiotrófica: análise dos instrumentos de avaliação. Rev Neurocienc. 2005 Jan-Mar; 13(1):21-7. doi:10.4181/RNC.2005.13.21.

13. Miller RG, Mitchell JD, Lyon M, Moore DH. Riluzole for amyotrophic lateral sclerosis (ALS)/motor neuron disease (MND). Cochrane Database Syst Rev. 2002; (2):CD001447. doi: 10.1002/14651858.CD001447.

14. Tiirola A, Korhonen T, Surakka T, Lehto JT. End-of-Life Care of Patients With Amyotrophic Lateral Sclerosis and Other Nonmalignant Diseases. Am J Hosp Palliat Care. 2017 Mar ;34(2):154-9. doi: 10.1177/1049909115610078.

15. Marin B, Boumédiene F, Logroscino G, Couratier P, Babron MC, Leutenegger $A L$, et al. Variation in world wide incidence of amyotrophic lateral sclerosis: a meta-analysis. Int J Epidemiol. 2017 Feb; 46(1):57-74. doi: 10.1093/ije/dyw061.

16. Demetriou CA, Hadjivasiliou PM, Kleopa KA, Christou YP, Leonidou E, Kyriakides T, et al. Epidemiology of Amyotrophic Lateral Sclerosis in the Republic of Cyprus: a 25-year retrospective study. Neuroepidemiology. 2017;48(1-2):7985. doi: 10.1159/000477126.

17. Rosenbohm A, Peter RS, Erhardt S, Lulé D, Rothenbacher D, Ludolph AC, et al. Epidemiology of amyotrophic lateral sclerosis in southern Germany. J Neurol. 2017 Apr; 264(4):749-57. doi: 10.1007/s00415-017-8413-3.

18. Pérez AM, Schiava M, Melcom M, Rodríguez G, Gargiulo G, Bettini M, et al. Estudio epidemiológico multicéntrico sobre esclerosis lateral amiotrófica en la Ciudad de Buenos Aires. Neurología Argentina. 2017; 9(4): 225-30. 
doi:10.1016/j.neuarg.2017.07.004

19. Arthur KC, Calvo A, Price TR, Geiger JT, Chiò A, Traynor BJ. Projected increase in amyotrophic lateral sclerosis from 2015 to 2040. Nat Commun. 2016; 7:12408. doi: $10.1038 /$ ncomms12408.

20. Prado LG, Bicalho IC, Vidigal-Lopes M, Ferreira CJ, Mageste Barbosa LS, Gomez RS, et al. Amyotrophic lateral sclerosis in Brazil: Case series and review of the Brazilian literature. Amyotroph Lateral Scler Frontotemporal Degener. 2016; 17(3-4):282-8. doi: 10.3109/21678421.2016.1143011.

21. Golby R, Poirier B, Fabros M, Cragg JJ, Yousefi M, Cashman N. Five-year incidence of amyotrophic lateral sclerosis in British Columbia (2010-2015). Can J Neurol Sci. 2016 Nov; 43(6):791-5. doi: 10.1017/cjn.2016.280.

22. Pradas J, Puig T, Rojas-García R, Viguera ML, Gich I, Logroscino G. Amyotrophic lateral sclerosis in Catalonia: a population based study. Amyotroph Lateral Scler Frontotemporal Degener. 2013 May; 14(4): 278-83. doi: 10.3109/21678421.2012.749915.

23. Chieia MA, Oliveira ASB, Silva HCA, Gabbai AA. Amyotrophic lateral sclerosis: considerations on diagnostic criteria. Arq. Neuro-Psiquiatr. $2010 \mathrm{Dec}$; 68(6): 837-842. doi: http://dx.doi.org/10.1590/S0004-282X2010000600002.
24. LI HF; WU ZY. Genotype-phenotype correlations of amyotrophic lateral sclerosis. Transl Neurodegener. 2016 Feb; 5: 3. doi: 10.1186/s40035-016-00508.

25. Paganoni S, Karam C, Joyce N, Bedlack R, Carter GT. Comprehensive rehabilitative care across the spectrum of amyotrophic lateral sclerosis. NeuroRehabilitation, 2015; 37(1):53-68. doi: 10.3233/NRE-151240.

26. Marin B, Bianchi E, Pupillo E, Lunetta C, Tremolizzo L, Logroscino G, et al. Non-self-sufficiency as a primary outcome measure in ALS trials. Amyotrophic Lateral SclerFrontotemporal Degener. 2015; 17(1-2), 77-84. doi: 10.3109/21678421.2015.1074704.

27. Kehyayan V, Korngut L, Jetté N, Hirdes JP. Profile of patients with amyotrophic lateral sclerosis across continuum of care. Can J Neurol Sci. 2014 Mar; 41(2): 246-252.

28. Andrews SC, Pavlis A, Staios M, Fisher F. Which behaviours? Identifying the most common and burdensome behaviour changes in amyotrophic lateral sclerosis. Psychol Health Med. 2017 Apr; 22(4):483-492. doi: 10.1080/13548506.2016.1164871.

\section{Como citar este artigo/How to cite this article:}

Silva LP, Gusmão CA, Pithon KR, Gomes TBP, Pinto EP Júnior. Esclerose lateral amiotrófica: descrição de aspectos clínicos e funcionais de uma série de casos numa região de saúde do nordeste do Brasil. J Health Biol Sci. 2018 Jul-Set; 6(3):293-298. 